\title{
Surveying Adjustment Datum and Relative Deformation Accuracy Analysis
}

\section{G.L. Chen ${ }^{1}$, X. Meng ${ }^{2 *}$, L.B. Yao ${ }^{3}$}

In the surveying adjustment, unknown parameters are normally different from the direct observations, but the elements related to these direct observations. In order to determine the unknown parameters, adequate known data should be provided and these necessarily needed known data are used to form the adjustment datum. Under different datum, different results will be obtained even with the same direct observations. However, in the practical adjustment calculation, the datum and its effect on the results are always ignored. In this paper, the adjustment datum is firstly discussed and defined as datum equations. Then an adjustment method based on the datum equations and the least squares is presented. This method is a generic one, not only suiting for the case in an ordinary datum but also in the gravity center datum or a quasi-datum, and can be easily used to analyze different deformations. Based on this method, the transformation between different reference frames is derived. It shows that the calculation results, deformation and positioning accuracy under one kind of datum are relative and generic. A case study is further introduced and used to test this new method. Based on the case study, the conclusions are reached. It is found that the relative positional root mean square error of each point becomes bigger as the distance between the point and the datum becomes longer and the relative deformation offsets under different kinds of datum are helpful for the reliable deformation analysis.

Keywords: Surveying adjustment, Datum equations, Relative position error, Relative deformation

\section{Introduction}

In the surveying adjustment, the unknown parameters are usually not the direct observations, but the elements related to these observations. For example, the observations are the values of direction and distance in a plane control network whilst the unknown parameters are the plane coordinates of the control points. In order to determine the unknown parameters, enough known data should be provided. This known data is necessary and called the adjustment datum. When the rank of the observation equation's coefficient matrix is less than the number of unknown parameters this is called a rank deficient problem in the adjustment [6].

The $n$-dimensional $(n=1 \sim 4)$ space has 1 scale datum, $n$ position datums and $n(n-1) / 2$ azimuth (direction) datums. That is to say the number of datums for one-dimensional space (height network) is 2 ( 1 scale datum and 1 position datum), for two-dimensional space (plane network) the number is 4 ( 1 scale datum, 2 position datums and 1 direction datum), for three-dimensional space the datum number is 7, and for four-dimensional space is 11 [2],[5],[10].

It should be pointed out that we can obtain the coordinates of the unknown points or deformations and their accuracy (variance matrix), which are coordinates and variance matrix under a given

${ }^{1}$ China University of Mining and Technology, Jiangsu, China

${ }^{2}$ The University of Nottingham, Nottingham UK

${ }^{3}$ Tongji University, Shanghai, China

*Corresponding author, email Xiaolin.meng@ nottingham.ac.uk 
relative datum from the adjustment for a control network or a monitoring network, according to a set of direct observations (direction, distance, GPS baseline vector, etc.) [7]. Under different datums, different results will be obtained with the same observations. However, in the practical adjustment calculation, the datum and its effect on the adjustment results are always omitted. The issue on measurement datum was emphasized in many studies [3],[6],[12].

In this paper, adjustment datum is firstly discussed and defined as datum equations. Then a new adjustment method based on the datum equations and the least squares is presented. This is a generic method and makes it easy to analyze the relative deformations among the monitoring stations [8],[9].

The adjustment datum has clear analytical relationship with the relative positional accuracy. [4] and [11] have outlined the complete definition of relative positional accuracy, which explains that it is not correct to understand the relative positional error as a mean square error of the coordinate differences of two points. In this paper, the transformational formula between different datums can be deduced by the method based on datum equations. The significance of the relative solutions, deformation, and positional accuracy attached to a certain datum are later explained.

\section{The datum and datum equations of the surveying adjustment}

Eq. 1 is the observation equation for surveying adjustment.

$$
\boldsymbol{L}+\boldsymbol{V}=f(\hat{\boldsymbol{X}})
$$

The Linearized error equation of Eq.1 can be expressed as follows:

$$
\boldsymbol{V}=\boldsymbol{A} \delta \hat{\boldsymbol{X}}-\boldsymbol{l}
$$

Where $L$ and $V$ are the observations and their corrections, $\hat{X}$ is $t$ unknown parameters, $P$ is a weight matrix of the observations, and $\delta \hat{\boldsymbol{X}}$ is a vector containing corrections to $\hat{\boldsymbol{X}}$. For a control network in a geometric space, it contains all the coordinates of the unknown points and other unknown parameters to form the adjustment datum. $A$ is the coefficient matrix. It is a rank deficient matrix and the rank can be estimated with $\operatorname{Rank}(\boldsymbol{A})=t-d$. The rank deficiency $d$ is equal to the number of the required datums and the relationship among the parameters is expressed as follows:

$$
\hat{\boldsymbol{X}}=\boldsymbol{X}^{0}+\delta \hat{\boldsymbol{X}} \quad \boldsymbol{l}=\boldsymbol{L}-f\left(\boldsymbol{X}^{0}\right)
$$

In order to get the unknown parameters $\delta \hat{X}$, we must select a set of datums, which means the parameters should be solved under the selected datums. Because all of the datums have a functional relationship with the unknown parameters, we can build an equation for each datum. For example, for the plane control network we can establish the following equations if we select points $P_{1}, P_{2}$ to form the adjustment datum:

$$
\left\{\begin{array}{l}
\delta \hat{X}_{1}=0 \\
\delta \hat{Y}_{1}=0 \\
\delta \hat{X}_{2}=0 \\
\delta \hat{Y}_{2}=0
\end{array}\right.
$$

We can also select $P_{1}$ as a fixed position, the length $S_{23}$ between points $P_{2}, P_{3}$ as the scale datum, and the azimuth $\alpha_{45}$ of $P_{4}, P_{5}$ as the direction datum. Then the new datum formed is as follows: 


$$
\left\{\begin{array}{l}
\delta \hat{X}_{1}=0 \\
\delta \hat{Y}_{1}=0 \\
\sqrt{\left(\hat{X}_{3}-\hat{X}_{2}\right)^{2}+\left(\hat{Y}_{3}-\hat{Y}_{2}\right)^{2}}-S_{23}=0 \\
\operatorname{arctg}\left(\left(\hat{Y}_{4}-\hat{Y}_{3}\right) /\left(\hat{X}_{4}-\hat{X}_{3}\right)\right)-\alpha_{45}=0
\end{array}\right.
$$

To make the nonlinear function above linearized and express them in a matrix form as:

$$
\boldsymbol{G}_{k}^{\mathrm{T}} \delta \hat{\boldsymbol{X}}+\boldsymbol{W}_{k}=0
$$

These three expressions can all be called datum equations. The coefficient matrix $\boldsymbol{G}_{k}^{\mathrm{T}}$ of the datum Eq. 4 is a full rank matrix whose rank should be equal to the number of datums $d: \operatorname{Rank}\left(\boldsymbol{G}_{k}^{\mathrm{T}}\right)=d$ and $\operatorname{Rank}\left(\boldsymbol{A}^{\mathrm{T}}, \boldsymbol{G}_{k}\right)=t$.

As can be seen from above derivations, each set of datums corresponds to a group of datum equations. If we select a group of equations, their coefficient matrix is full ranked and their rank is equal to the required datum number $d$. In this case we can also regard them as the datum equations.

Assume that there is an equation:

$$
\boldsymbol{G}_{k}^{\mathrm{T}} \delta \hat{\boldsymbol{X}}=0
$$

The rank of $\boldsymbol{G}$ is $\operatorname{Rank}(\boldsymbol{G})=d$ and it meets the condition $\boldsymbol{A} \boldsymbol{G}=0$. Select the equation as a datum equation - we generally call it a gravity center datum equation. For a plane control network with coordinates as unknown parameters, generally we can set $\boldsymbol{G}$ as:

$$
\boldsymbol{G}^{\mathrm{T}}=\left[\begin{array}{ccccccc}
1 & 0 & 1 & 0 & \cdots & 1 & 0 \\
0 & 1 & 0 & 1 & \cdots & 0 & 1 \\
X_{1}^{0} & Y_{1}^{0} & X_{2}^{0} & Y_{2}^{0} & \cdots & X_{m}^{0} & Y_{m}^{0} \\
-Y_{1}^{0} & X_{1}^{0} & -Y_{2}^{0} & X_{2}^{0} & \cdots & -Y_{m}^{0} & X_{m}^{0}
\end{array}\right]
$$

The solution given under the gravity center datum is also the solution of a rank deficient adjustment. The geometric meaning of gravity center datum is: position datum is fixed to the average values of each point's coordinates (gravity coordinate); the scale datum is fixed to the weighted average of the ratios of the adjusted distances for each point to the gravity center over their initial values; and the directional datum is fixed to the weighted average azimuth value of each point to the gravity center.

Let $\boldsymbol{G}^{\mathrm{T}}$ divided into two parts and set $\boldsymbol{G}^{\mathrm{T}}=\left[\begin{array}{ll}G_{1}^{\mathrm{T}} & G_{2}^{\mathrm{T}}\end{array}\right]$ :

$$
\begin{aligned}
\boldsymbol{G}_{k}^{\mathrm{T}} & =\left[\begin{array}{lllccccc}
0 & \mathrm{G}_{2}^{\mathrm{T}}
\end{array}\right] \\
& =\left[\begin{array}{cccccccc}
0 & \cdots & 0 & 1 & 0 & \cdots & 1 & 0 \\
0 & \cdots & 0 & 0 & 1 & \cdots & 0 & 1 \\
0 & \cdots & 0 & X_{P}^{0} & Y_{P}^{0} & \cdots & X_{m}^{0} & Y_{m}^{0} \\
0 & \cdots & 0 & -Y_{P}^{0} & X_{P}^{0} & \cdots & -Y_{m}^{0} & X_{m}^{0}
\end{array}\right]
\end{aligned}
$$

Set the datum equation:

$$
G_{2}^{\mathrm{T}} \delta \hat{X}_{2}=0
$$

This datum is said to fix the gravity centre of some points as the overall datum, which is also called the quasi-stable datum. 


\section{The solution based on datum equations}

\section{Normal equation and its solution}

In order to obtain the least squares solution $\delta \hat{\boldsymbol{X}}$ under the selected datum, we can use datum data to eliminate $d$ datum parameters in $\delta \hat{\boldsymbol{X}}$. This can make the design matrix $\boldsymbol{A}$ be a full rank matrix and then we can get the solution with the least squares adjustment. However it is easy to overlook the significance of the datum. Therefore, it is necessary to use the following method.

The datum equations show the functional relationship among the unknown parameters, and they form the conditional equations. We can get the normal equations under parameter adjustment with constraints through combining Eq. 2 and Eq. 4:

$$
\left\{\begin{array}{l}
\boldsymbol{A}^{\mathrm{T}} \boldsymbol{P A} \delta \hat{\boldsymbol{X}}+\boldsymbol{G}_{k} \boldsymbol{K}-\boldsymbol{A}^{\mathrm{T}} \boldsymbol{P l}=0 \\
\boldsymbol{G}_{k}^{\mathrm{T}} \delta \hat{\boldsymbol{X}}+\boldsymbol{W}_{k}=0
\end{array}\right.
$$

where $\boldsymbol{K}$ is the connection number vector of the datum equations.

Left multiply the first equation in (7) with $\boldsymbol{G}^{\mathrm{T}}$, we get:

$$
\boldsymbol{G}^{\mathrm{T}} \boldsymbol{A}^{\mathrm{T}} \boldsymbol{P A} \delta \hat{\boldsymbol{X}}+\boldsymbol{G}^{\mathrm{T}} \boldsymbol{G}_{k} \boldsymbol{K}-\boldsymbol{G}^{\mathrm{T}} \boldsymbol{A}^{\mathrm{T}} \boldsymbol{P l}=0
$$

With $\quad \boldsymbol{A} \boldsymbol{G}=0$ and the rank of $\boldsymbol{G}^{\mathrm{T}}$

$$
\begin{aligned}
& \operatorname{Rank}\left(\boldsymbol{G}^{\mathrm{T}}\right)=\operatorname{Rank}\left(\boldsymbol{G}_{k}^{\mathrm{T}}\right)=\operatorname{Rank}\left(\boldsymbol{G}^{\mathrm{T}} \boldsymbol{G}_{k}\right)=d \\
& \operatorname{Rank}\left(\boldsymbol{A}^{\mathrm{T}} \boldsymbol{P A}\right)=\operatorname{Rank}(\boldsymbol{A})=t-d
\end{aligned}
$$

We get $\boldsymbol{K}=0$.

Left multiply the second equation in (7) with $\boldsymbol{G}_{k}$ and add it to the first equation, we get

$$
\left(\boldsymbol{A}^{\mathrm{T}} \boldsymbol{P A}+\boldsymbol{G}_{k} \boldsymbol{G}_{k}^{\mathrm{T}}\right) \delta \hat{\boldsymbol{X}}-\left(\boldsymbol{A}^{\mathrm{T}} \boldsymbol{P l}-\boldsymbol{G}_{k} \boldsymbol{W}_{k}\right)=0
$$

Since $\operatorname{Rank}\left(\boldsymbol{A}^{\mathrm{T}} \boldsymbol{G}_{k}\right)=t$, we get:

$$
\operatorname{Rank}\left(\boldsymbol{A}^{\mathrm{T}} \boldsymbol{P A}+\boldsymbol{G}_{k} \boldsymbol{G}_{k}^{\mathrm{T}}\right)=t
$$

Eq. (8) is a full rank equation - it could obtain the least squares solution based on the datum equation (4):

$$
\delta \hat{\boldsymbol{X}}_{k}=\boldsymbol{Q}_{k}\left(\boldsymbol{A}^{\mathrm{T}} \boldsymbol{P} \boldsymbol{l}-\boldsymbol{G}_{k} \boldsymbol{W}_{k}\right)
$$

Where $\boldsymbol{Q}_{k}=\left(\boldsymbol{A}^{\mathrm{T}} \boldsymbol{P A}+\boldsymbol{G}_{k} \boldsymbol{G}_{k}^{\mathrm{T}}\right)^{-1}$, the subscript $k$ represents used here is similar as that in Eq.(4). Eq.(8) is also called the normal equations, corresponding to the datum Eq. (4).

\section{Co-efficient factor of the unknown parameters}

Applying the covariance propagation law to Eq. (9), the co-efficient factor of $\delta \hat{\boldsymbol{X}}$ could be estimated as follows:

$$
\boldsymbol{Q}_{\hat{\boldsymbol{x}}_{k}}=\boldsymbol{Q}_{k} \boldsymbol{A}^{\mathrm{T}} \boldsymbol{P} \boldsymbol{A} \boldsymbol{Q}_{k}
$$

Since $\boldsymbol{Q}_{k}\left(\boldsymbol{A}^{\mathrm{T}} \boldsymbol{P A}+\boldsymbol{G}_{k} \boldsymbol{G}_{k}^{\mathrm{T}}\right)=\boldsymbol{I}$ and $\boldsymbol{A} \boldsymbol{G}=0$ thus $\boldsymbol{Q}_{k} \boldsymbol{A}^{\mathrm{T}} \boldsymbol{P A}=\boldsymbol{Q}_{k}\left(\boldsymbol{A}^{\mathrm{T}} \boldsymbol{P A}+\boldsymbol{G}_{k} \boldsymbol{G}_{k}^{\mathrm{T}}-\boldsymbol{G}_{k} \boldsymbol{G}_{k}^{\mathrm{T}}\right)=\boldsymbol{I}-\boldsymbol{Q}_{k} \boldsymbol{G}_{k} \boldsymbol{G}_{k}^{\mathrm{T}}$

$$
\boldsymbol{Q}_{k} \boldsymbol{G}_{k}=\boldsymbol{Q}\left(\boldsymbol{A}^{\mathrm{T}} \boldsymbol{P} \boldsymbol{A}+\boldsymbol{G} \boldsymbol{G}_{k}^{\mathrm{T}} \boldsymbol{G}_{k} \boldsymbol{G} \boldsymbol{G}_{k}^{-}\right)_{\bar{k}}^{\frac{1}{k}} \boldsymbol{G} \boldsymbol{G}\left(\boldsymbol{G}^{\mathrm{T}-}{ }_{k}\right.
$$


So,

$$
\boldsymbol{Q}_{\hat{x}_{k}}=\boldsymbol{Q}_{k}-\boldsymbol{Q} \boldsymbol{G} \boldsymbol{G}^{\mathrm{T}} \boldsymbol{Q}_{k}=\boldsymbol{Q}-\boldsymbol{G}\left(\boldsymbol{G} \boldsymbol{G}_{k} \boldsymbol{G}_{\boldsymbol{G}} \boldsymbol{G}^{\mathrm{T}}-\boldsymbol{G}_{k}^{1}\right.
$$

The co-efficient factors of corrected observations $\hat{L}$ and their corrections $V$ could also be calculated:

$$
\left\{\begin{array}{l}
\boldsymbol{Q}_{\hat{L}}=\boldsymbol{A} \boldsymbol{Q}_{\hat{\boldsymbol{X}}_{k}} \boldsymbol{A}^{\mathrm{T}}=\boldsymbol{A} \boldsymbol{Q}_{k} \boldsymbol{A}^{\mathrm{T}} \\
\boldsymbol{Q}_{\boldsymbol{V}}=\left(\boldsymbol{A} \boldsymbol{Q}_{k} \boldsymbol{A}^{\mathrm{T}} \boldsymbol{P}-\boldsymbol{I}\right) \boldsymbol{Q}\left(\boldsymbol{A} \boldsymbol{Q}_{k} \boldsymbol{A}^{\mathrm{T}} \boldsymbol{P}-\boldsymbol{I}\right)^{\mathrm{T}}=\boldsymbol{Q}-\boldsymbol{A} \boldsymbol{Q}_{k} \boldsymbol{A}^{\mathrm{T}}
\end{array}\right.
$$

\section{Unit weight estimate}

Since

$$
\left.E\left(\boldsymbol{V}^{\mathrm{T}} \boldsymbol{P} \boldsymbol{V}\right)=t \boldsymbol{R} \quad \boldsymbol{E} \boldsymbol{V}^{\mathrm{T}} \neq\right) \boldsymbol{P} r_{V}\left(D \varnothing_{0}\right)^{2} \boldsymbol{P} \boldsymbol{Q}_{v} r(
$$

Taking the second expression of Eq. (13) into Eq.(14) and consider Eq. (11), we have:

$$
\begin{gathered}
E\left(\boldsymbol{V}^{\mathrm{T}} \boldsymbol{P} \boldsymbol{V}\right)=\sigma_{0}^{2} \operatorname{tr}\left(\boldsymbol{P}\left(\boldsymbol{Q}-\boldsymbol{A} \boldsymbol{Q}_{k} \boldsymbol{A}^{\mathrm{T}}\right)\right)=\sigma_{0}^{2} \operatorname{tr}\left(\boldsymbol{P} \boldsymbol{Q}-\boldsymbol{Q}_{k} \boldsymbol{A}^{\mathrm{T}} \boldsymbol{P A}\right) \\
\quad=\sigma_{0}^{2} \operatorname{tr}\left(\boldsymbol{P} \boldsymbol{Q}-\boldsymbol{Q}_{k} \boldsymbol{Q}_{k}^{-1}+\boldsymbol{G}\left(\boldsymbol{G}_{k}^{\mathrm{T}} \boldsymbol{G}\right)^{-1} \boldsymbol{G}_{k}^{\mathrm{T}}\right)=\sigma_{0}^{2}(n-t+d)
\end{gathered}
$$

Then the estimation formula of unit weight variance can be calculated as follows:

$$
\hat{\sigma}_{0}^{2}=\frac{1}{n-t+d} \boldsymbol{V}^{\mathrm{T}} \boldsymbol{P} \boldsymbol{V}
$$

\section{Solutions with gravity center datum and quasi-stable datum}

When the gravity centre datum is used (refer to Eq. (5)), the solution of the least squares can be expressed as Eq. (16):

$$
\left\{\begin{array}{l}
\delta \hat{\boldsymbol{X}}_{g}=\left(\boldsymbol{A}^{\mathrm{T}} \boldsymbol{P A}+\boldsymbol{G} \boldsymbol{G}\right)^{-} \boldsymbol{A} \boldsymbol{P} \boldsymbol{L}=\boldsymbol{Q}_{g} \boldsymbol{A} \boldsymbol{P} \boldsymbol{l}^{\mathrm{T}} \\
\boldsymbol{Q}_{\hat{\boldsymbol{X}}}=\boldsymbol{Q}_{g}-\boldsymbol{G}\left(\boldsymbol{G}^{\mathrm{T}} \boldsymbol{G}\right)^{-}(\boldsymbol{G} \boldsymbol{G})^{-} \boldsymbol{G}^{1}
\end{array}\right.
$$

Eq. (16) is a rank deficient solution. It can be proved that the solution (rank deficient solution) based on the gravity center datum has the following characteristics:

(i) $\delta \hat{\boldsymbol{X}}_{g}^{\mathrm{T}} \delta \hat{\boldsymbol{X}}_{g}=\min$

(ii) $\operatorname{Tr}\left(\boldsymbol{Q}_{\hat{x}_{s}}\right)=\min$

(iii) Unbiased when $\boldsymbol{G}^{\mathrm{T}} \delta \hat{\boldsymbol{X}}=0$.

When the quasi-stable datum is used, as described with Eq. (6), its observation and normal equations could be expressed as follows:

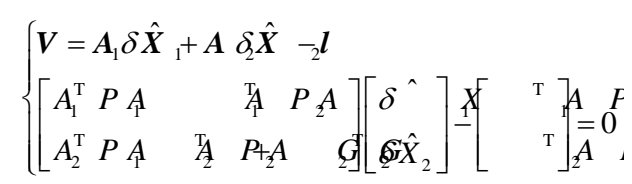

From above discussion it can be found that once the datums are decided the unknown parameters could be estimated with Eqs. (9) and (12). These above methods are not only suitable for the solutions under the general datums, but also fit for the solutions under both the gravity center datum and the quasi-stable datum.

\section{Relative deformation and its positional accuracy}

Suppose the datum expressed by Eq. (4) have a solution $\delta \hat{\boldsymbol{X}}_{k}$ and its corresponding covariance factor 
is $\boldsymbol{Q}_{\hat{X}_{k}}$. If there is another datum expressed as Eq. (18):

$$
\boldsymbol{G}_{j}^{\mathrm{T}} \delta \hat{\boldsymbol{X}}_{j}+\boldsymbol{W} \overline{\bar{j}} 0
$$

The solution of the datum Eq. (18) could also be calculated using a similar approach as introduced above:

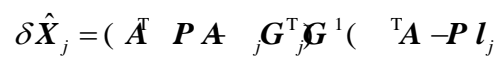

Next, to derive the relationship between $\delta \hat{\boldsymbol{X}}_{j}, \boldsymbol{Q}_{\hat{x}_{j}}$ and $\delta \hat{\boldsymbol{X}}_{k}, \boldsymbol{Q}_{\hat{\boldsymbol{X}}_{k}}$. Transform the above expression to:

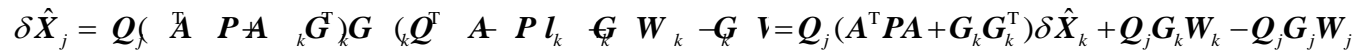

$$
\begin{aligned}
& =\boldsymbol{Q}_{j} \boldsymbol{A}^{\mathrm{T}} \boldsymbol{P} \boldsymbol{A} \delta \hat{\boldsymbol{X}}_{k}+\boldsymbol{Q}_{j} \boldsymbol{G}_{k}\left(\boldsymbol{G}_{k}^{\mathrm{T}} \delta \hat{\boldsymbol{X}}_{k}+\boldsymbol{W}_{k}\right)-\boldsymbol{Q}_{j} \boldsymbol{G}_{j} \boldsymbol{W}_{j}
\end{aligned}
$$

Considering Eq. (7) and the relationship of Eq. (11), then:

$$
\delta \hat{\boldsymbol{X}}_{j}=\left(\boldsymbol{I}-\boldsymbol{Q} \boldsymbol{G} \boldsymbol{G}^{\mathrm{T}}\right)_{j} \hat{\boldsymbol{X}}-\boldsymbol{Q} \boldsymbol{G} \boldsymbol{W}={ }_{\bar{j}}\left[\boldsymbol{I}_{j}-\boldsymbol{G} \boldsymbol{G} \boldsymbol{G}^{-} \boldsymbol{G}_{j}^{\mathrm{T}}\right]^{\mathrm{T}} \hat{\boldsymbol{x}}_{j} \boldsymbol{G} \boldsymbol{G}\left(\boldsymbol{G}^{\mathrm{T}} \boldsymbol{W}\right)^{\prime}
$$

According to the covariance propagation law, then:

$$
\boldsymbol{Q}_{\hat{\boldsymbol{x}}_{j}}=\left(\boldsymbol{I}-\boldsymbol{Q}_{j} \boldsymbol{G} \boldsymbol{G}^{\mathrm{T}} \boldsymbol{Q}_{\hat{\boldsymbol{x}}_{k}} \boldsymbol{I}\left(-\boldsymbol{Q} \boldsymbol{G}_{j} \boldsymbol{G}_{j}^{\mathrm{T}} \quad \mathrm{z}_{j}\left[\boldsymbol{I}-\boldsymbol{G} \boldsymbol{G}\left(\boldsymbol{G}^{\mathrm{T}-}{ }_{j} \boldsymbol{G}\right)^{1}\right] \boldsymbol{Q}_{\dot{x}^{\mathrm{T}} j_{k}}^{\mathrm{T}}\left[\boldsymbol{I}-\boldsymbol{G} \boldsymbol{G} \boldsymbol{G}{ }^{-\mathrm{T}} \boldsymbol{G}\right)\right]^{\mathrm{T}_{\mathrm{T}}}\right.
$$

Eqs. (20) and (21) represent the relationship between $\delta \hat{\boldsymbol{X}}_{j}, \boldsymbol{Q}_{\hat{\boldsymbol{X}}_{i}}$ and $\delta \hat{\boldsymbol{X}}_{k}, \boldsymbol{Q}_{\hat{\boldsymbol{X}}_{k}}$, and they can be used to transform solutions obtained under different datums.

It is not difficult to understand that to an adjustment problem using of different datums will produce different solutions with different accuracies. Those different datums and their corresponding covariance factors could be transformed using Eqs. (20) and (21). In other words, the solutions and accuracies of an adjustment problem are only meaningful when attached to a certain datum. As for a plane control network or 3-dimensional control network, using Eqs. (20) and (21) the solution and its corresponding positional accuracy under the one datum could be expressed as the results in another datum. This kind of relative positional accuracy is generalized one. Compared with the relative positional accuracy based on the coordinate difference of two points, this expression has a more comprehensive meaning.

Suppose $\delta \hat{\boldsymbol{X}}_{k}$ and $Q_{\hat{\boldsymbol{x}}_{k}}$ are the solution and associated covariance factor attained based on one datum in a plane monitoring network, the relative deformation and relative positional accuracy based on another datum could be obtained according to Eqs. (20) and (21). If there are three points $P_{1}, P_{2}, P_{3}$ in a monitoring network and named $P_{1}, P_{2}$ to form the datum points, and the deformation value and relative positional error of $P_{3}$ relative to $P_{1}, P_{2}$ could be determined with Eqs. (20) and (21). The same process can be used for $P_{2}$ when points $P_{1}, P_{3}$ are used the datum and so on. We call them a three-point relative deformation value and three-point relative positional accuracy.

\section{Example}


There is a GPS deformation network shown as Figure 1. If the plane coordinates based on a datum in the previous period had been determined, we want to analyze the deformation according to the new GPS observations acquired. Take the plane coordinates from G06 and G08 as the datum we could obtain the deformation value and its accuracy of each point in this datum, and then using Eqs (20) and (21) to attain the results of each point by using any two points as a datum.

Fig. 1 A GPS deformation network

Table 1 lists the deformation values ( $\mathrm{dx}, \mathrm{dy}$ and $\mathrm{dz}$ ) and their corresponding accuracies (Mx, My and Mz) of points G03, G06, and G05, relative to any two datums. We could conclude from Table 1:

Table 1 Relative positional accuracy and deformation offset $(\mathrm{cm})$

\begin{tabular}{lllllllll}
\hline Point & $\mathrm{dx}$ & $\mathrm{dy}$ & $\mathrm{ds}$ & \multicolumn{2}{l}{ Datum point } & $\mathrm{Mx}$ & $\mathrm{My}$ & $\mathrm{Mp}$ \\
\hline $\mathrm{G} 03$ & 0.06 & -0.06 & 0.08 & $\mathrm{G} 04$ & $\mathrm{G} 02$ & 0.09 & 0.10 & 0.14 \\
& 0.20 & 0.23 & 0.30 & $\mathrm{G} 01$ & $\mathrm{G} 08$ & 0.12 & 0.15 & 0.19 \\
& -2.19 & -1.04 & 2.42 & $\mathrm{G} 05$ & $\mathrm{G} 01$ & 0.12 & 0.17 & 0.21 \\
& 1.72 & -3.49 & 3.89 & $\mathrm{G} 05$ & $\mathrm{G} 08$ & 0.23 & 0.18 & 0.29 \\
& 0.37 & -0.21 & 0.43 & $\mathrm{G} 09$ & $\mathrm{G} 07$ & 0.49 & 0.50 & 0.71 \\
G06 & -0.08 & -0.01 & 0.08 & G07 & G09 & 0.09 & 0.11 & 0.14 \\
& -0.67 & -0.75 & 1.01 & G02 & G08 & 0.31 & 0.37 & 0.48 \\
& -0.59 & -0.83 & 1.02 & G01 & G08 & 0.35 & 0.39 & 0.53 \\
& -1.64 & 8.11 & 8.27 & G05 & G01 & 0.28 & 0.29 & 0.40 \\
& -3.88 & 14.24 & 14.76 & G05 & G08 & 0.57 & 0.21 & 0.61 \\
& -2.15 & -0.48 & 2.20 & G03 & G04 & 0.60 & 0.46 & 0.75 \\
& 3.44 & -2.47 & 4.23 & G07 & G01 & 0.20 & 0.18 & 0.27 \\
& 2.90 & -2.71 & 3.97 & G04 & G08 & 0.15 & 0.23 & 0.28 \\
& 2.75 & -3.02 & 4.08 & G03 & G02 & 0.37 & 0.25 & 0.45 \\
& 3.70 & -3.10 & 4.83 & G06 & G09 & 0.32 & 0.33 & 0.46
\end{tabular}

i). Normally, the further a point away from the datum points, the higher the relative positional root mean square error.

ii). In each kind of datum, point G05 shows a larger deformation value than the other points. However when G05 is not used as datum point, G03, G06 and other points show smaller deformations but when G05 is included all of these points exhibit larger deformations. This phenomenon illustrates that G05 has a deformations of more than $4 \mathrm{~cm}$.

\section{Conclusions}

From the above study the following conclusions can be drawn:

1). Datum data could be represented by the datum equations, and a set of datum equations could represent a set of datums.

2). Finding the least squares adjustment solution using datum equations is a universal approach. It is not only suitable for general datums, but also suitable for the gravity center datum and the quasi-stable datum.

3). We could derive the transform formula to solve the solution of different datums by this 
means, and it is easy to analyze the relevant problem.

4). Relative solution, relative deformation and relative positional accuracy are generalized and comprehensive.

5). Utilizing the concept of relative deformation and relative positional accuracy could make analysis of deformation much easier and more reliable.

\section{Acknowledgement}

Professor Dajie Liu is remembered for his preparation of the first draft paper and his guidance to the second and third authors of this paper when they were his $\mathrm{PhD}$ students of Tongji University in Shanghai, China. This research project was jointly sponsored by National Natural Science Foundation of China (No.41371423).

\section{References}

1. Chen J.Y, 2003. Thinking on the Establishment of Modern Geodetic Datum of China. Geomatics and Information Science of Wuhan University, 28(5) : 1-6.

2. Cui X.Z, Yu Z.T, Tao B.Z and Liu.D.J, 2009. Generalized Surveying Adjustment. Wuhan University Press.

3. Huang L.R, 2001. Reference Frame and Its Advisability in Deformation Analysis of GPS Observation Results. Acta Geodaetica et Cartographica Sinica, (1):16-20.

4. Liu D.J, 1984. Relative Positional Accuracy of Plane Control Network. Acta Geodaetica et Cartographica Sinica, (3):182-191

5. Liu D.J and Feng Y.M, 2003. Adjustment Datum for Dynamic Geodesy. Geomatics and information Science of Wuhan University, 28(5):69-72.

6. Lu T.D, Tao B.Z and Zhou S.J, 2007. Characteristics of Matrix SVD and Its Applications to Rank Deficiency Free Network Adjustment. Journal of Geodesy and Geodynamics, 27(5):63-67.

7. Tang W.M, Shi C and Meng X.L, 2011. Single Epoch Ambiguity Resolution Algorithms for Single Frequency GPS with Baseline Length. Survey Review, 43(322):322-332.

8. Wang L.Y and Xu C.J, 2011. Total Least Squares Adjustment with Weight Scaling Factor. Geomatics and Information Science of Wuhan University, 36(8):887-890.

9. Wang L.Y, Xu C.J and Lu T.D, 2010. Ridge Estimation Method in Ill-posed Weighted Total Least Squares Adjustment. Geomatics and Information Science of Wuhan University, 35(11):1346-1350.

10. Wu J.J, 2006. Research in Transformation Model Between Two Geodetic Coordinate Systems. Journal of Henan Polytechnic University(Natural Science), 25(5): 383-385.

11. Xu C.J and Liu D.J, 1990. The Broad Error Ellipsoid (Ellipse). Journal of Wuhan Technical University of Surveying and Mapping, (2):25-30.

12. Ye S.H and Huang C, 2000. Astro-geo dynamics. Shandong Science \& Technology Press. 